\title{
A Comprehensive Analysis of the MAGE Family as Prognostic and Diagnostic Markers for Hepatocellular Carcinoma
}

\section{Rong Li}

Guangdong Provincial Key Laboratory of Liver Disease Research, Guangdong province engineering laboratory for transplantation medicine, Third Affiliated Hospital of Sun Yat-sen University

\section{Jiao Gong}

Department of Laboratory Medicine, Third Affiliated Hospital of Sun Yat-sen University

\section{Cuicui Xiao}

Department of Anesthesiology, Cell-Gene therapy Translational Medicine Research Center, Third Affiliated Hospital of Sun Yat-sen University

\section{Shuguang Zhu}

Department of Hepatic Surgery and Liver Transplantation Center, The Third Affiliated Hospital of Sun Yat-sen University

\section{Zhongying Hu}

Guangdong provincial Key Laboratory of Liver Disease Research, Third Affiliated Hospital of Sun Yatsen University

\section{Jinliang Liang}

Guangdong Provincial Key Laboratory of Liver Disease Research, Third Affiliated Hospital of Sun Yatsen University

\section{Xuejiao Li}

Guangdong Provincial Key Laboratory of Liver Disease Research, Third Affiliated Hospital of Sun Yatsen University

\section{Xijing Yan}

Department of Hepatic Surgery and Liver Transplantation Center, Third Affiliated Hospital of Sun Yat-sen University

\section{Xijian Zhang}

Department of Hepatic Surgery and Liver Transplantation Center, Third Affiliated Hospital of Sun Yat-sen University

\section{Danyang Li}

Department of Infectious Diseases, Third Affiliated Hospital of Sun Yat-sen University

\section{Wei Liu}

Guangdong Provincial Key Laboratory of Liver Disease Research, Guangdong province engineering laboratory for transplantation medicine, Third Affiliated Hospital of Sun Yat-sen University 


\section{Yutian Chong}

Guangdong Provincial Key Laboratory of Liver Disease Research, Department of Infectious Diseases, Third Affiliated Hospital of Sun Yat-sen University

\section{Yusheng Jie ( $\nabla_{\text {jieyusheng@163.com ) }}$}

Third Affiliated Hospital of Sun Yat-Sen University https://orcid.org/0000-0003-3756-0653

\section{Primary research}

Keywords: MAGE family, Hepatocellular carcinoma, prognostic and diagnostic markers

Posted Date: July 14th, 2020

DOI: https://doi.org/10.21203/rs.3.rs-40996/v1

License: (c) (i) This work is licensed under a Creative Commons Attribution 4.0 International License. Read Full License

Version of Record: A version of this preprint was published at Genomics on November 1st, 2020. See the published version at https://doi.org/10.1016/j.ygeno.2020.09.026. 


\section{Abstract}

Background: The Melanoma Antigen Gene (MAGE) family is a large, highly conserved group of proteins that share a common MAGE homology domain. Multiple MAGEs are aberrantly expressed in a variety of cancers. However, the function of distinct MAGE genes in hepatocellular carcinoma (HCC) is largely unclear. Our study aimed to comprehensively analyze the MAGE family as prognostic and diagnostic markers for HCC.

Methods: In this research, all HCC data were obtained from NCBI GEO DataSets, The Cancer Genome Atlas (TCGA) and our clinical center. UALCAN was used to reveal the expression profile of distinct MAGEs in $\mathrm{HCC}$ and further evaluate the association between the expression of MAGEs and the clinicopathological characteristics of HCC patients, including clinical stage, tumor grade. Kaplan-Meier Plotter (KM-Plot) was used to evaluate the correlation between MAGEs expression and the overall survival (OS) of HCC patients. qRT-PCR was used to detection the expression levels of MAGEs in HCC samples. cBioPortal was used to analyze the genetic alterations in MAGEs and their associations with OS of HCC patients. Gene Set Variation Analysis (GSVA) algorithm was used to do functional enrichment analysis of MAGE genes in HCC to reveal the molecular mechanisms of MAGEs functioned in HCC.

Results: Our research showed that many MAGE genes were dysregulated in HCC and most of them were highly expressed. Among them, MAGEA1DMAGEC2ロMAGED1DMAGED2ロMAGEF1 and MAGEL2 were significantly correlated with clinical stage and differentiation of HCC patients. MAGED1DMAGED2ם

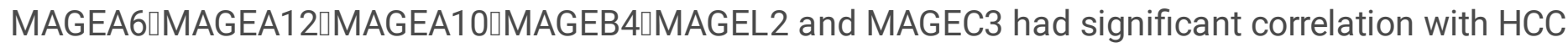
prognosis. Further functional enrichment analysis suggested the dysregulated MAGEs may play important roles in signal transduction.

Conclusions: Taken together, our research revealed that multiple MAGEs were dysregulated in HCC and they might play important roles in the development of $\mathrm{HCC}$ and can be exploited as useful biomarkers for diagnosis and treatment for HCC.

\section{Background}

Primary liver cancer is one of the leading causes of cancer-related death worldwide with high incidence rate $(4.7 \%)$ and mortality rate $(8.2 \%)$. Hepatocellular carcinoma $(\mathrm{HCC})$ is the main type of primary liver cancer (comprising $75 \%-85 \%$ of cases)[1]. Approximately 750000 new cases are diagnosed with HCC and nearly 500000 deaths annually worldwide[2]. Due to the lack of obvious symptom in early stage and effective early diagnosis methods, only $30-40 \%$ of HCC patients can be performed curative resection. However, the prognosis of these patients are still poor, the five-year recurrence can reach up to $70 \%[3,4]$. The grim situation urges us to further elucidate the potential molecular mechanism underlying the occurrence and development of HCC, identify more efficient biomarkers for early diagnosis, prognosis prediction and find new targets to design more powerful therapeutic methods to improve the prognosis of HCC patients. 
The first Melanoma Antigen Gene (MAGE) protein was identified in a melanoma patient due to its strong tumor antigen properties in 1980s[5]. The subsequent studies and homology searches on MAGEs revealed that the MAGE family consists of about 60 members which are conserved in all eukaryotes and share a common MAGE homology domain[6, 7]. Based on the chromosomal location and expression pattern, the MAGE family members can be divided into two classes, type I MAGEs and type II MAGEs [8]. Type I MAGEs include the MAGE-A, MAGE-B and MAGE-C subfamily members which are located on the $X$ chromosome and mainly expressed in the testis and aberrantly expressed in tumor tissues. Type II MAGEs include the MAGE-D, MAGE-E, MAGE-F, MAGE-G, MAGE-H, MAGE-L and Necdin which are not restricted to the $X$ chromosome and expressed in a variety of tissues $[6,9,10]$.

Until now, multiple MAGEs have been reported to be dysregulated in a variety of cancers, including melanoma [11-13], non-small cell lung cancer [14, 15], colon cancer [16-18], glioma [19, 20], breast cancer $[21,22]$ and prostate cancer $[23,24]$. Further functional studies show that the aberrant expression of MAGEs correlated with worse clinical prognosis of tumor patients, including shorter overall survival (OS) time, shorter disease free survival (DFS) time, larger tumor size, metastasis and so on. The characteristics of MAGEs in tumors suggest them suitable to serve as targets for diagnosis and treatment in various cancers[7, 25].

Previous studies on the MAGEs in HCC also suggest that the aberrant expression of multiple MAGEs is significantly correlated with the clinical characteristics of HCC patients [26]. However, the MAGE family contains about 60 members and the expression profile and function of most MAGE members in HCC are unclear yet. In this research, we integrated and analyzed almost $600 \mathrm{HCC}$ patients' data from NCBI GEO DataSets, TCGA (The Cancer Genome Atlas) and our clinical center using Kaplan-Meier Plotter (KM-Plot), UALCAN, cBioPortal databases and Gene Set Variation Analysis (GSVA) algorithm to comprehensively evaluate the function and clinical significance of MAGEs in HCC. Our study revealed that multiple MAGEs were dysregulated in HCC and significantly correlated with clinicopathological parameters. Further functional enrichment analysis suggested the dysregulated MAGEs may play important roles in signal transduction which regulate the development of HCC. The characteristics of MAGEs in HCC suggest that they are suitable to serve as useful biomarkers to diagnosis and treatment HCC.

\section{Results}

\section{Expression profile of MAGE family in HCC}

We first analyzed the expression profile of MAGE genes in GSE14520 (obtained from NCBI GEO DataSets), a microarray data of HCC containing 225 tumor tissues and 220 adjacent non-tumor tissues.

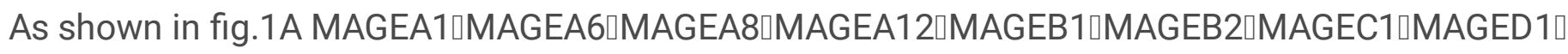
MAGED2 and MAGEF1 were significantly up-regulated in HCC tissues compared with non-tumor tissues.

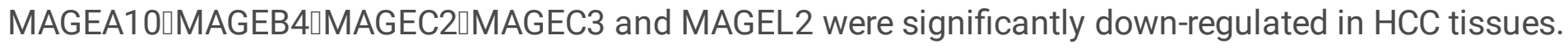
We further confirmed the expression of MAGE genes in HCC using TCGA data. As shown in fig.1B,

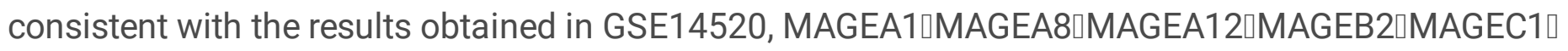


MAGED1DMAGED2 and MAGEF1 were significantly up-regulated in HCC tissues and MAGEL2 was significantly down-regulated in HCC tissues. In contrast to GSE14520, MAGEC2 was up-regulated in HCC tissues of TCGA. We speculated that this was related to the low expression of MAGEC2 in tissues. In

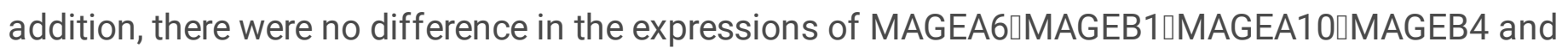
MAGEC3 between HCC tissues and non-tumor tissues in TCGA dataset, which may also be accounted for the low expression of those genes. Taken together, the above data indicated that multiple MAGE genes were dysregulated in $\mathrm{HCC}$ and most of them were highly expressed, suggesting that the dysregulated MAGEs may play important roles in the development of HCC.

\section{Correlation between the expression of MAGE genes and the clinicopathological parameters of HCC patients}

To further evaluate the roles of dysregulated MAGEs in HCC, we analyzed the association between MAGEs expression and clinical stage and differentiation of HCC patients with TCGA data using UALCAN (http://ualcan.path.uab.edu). As shown in fig.2A, the expression of MAGEA1DMAGEC2ロMAGED1ם MAGED2\MAGEF1 and MAGEL2 were significantly correlated with HCC patients' clinical stage. Among them, the expressions of MAGEA1DMAGEC2IMAGED1DMAGED2 and MAGEF1 were increased with the clinical stage, especially for of MAGED2 and MAGEF1. Another MAGE gene, MAGEL2, was decreased with clinical stage.

Fig.2B showed the correlation between MAGEs expression and HCC tumor grade. Similar to clinical stage,

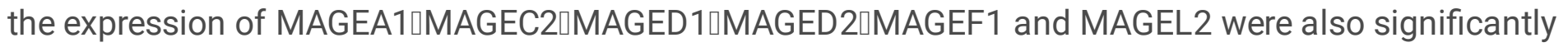

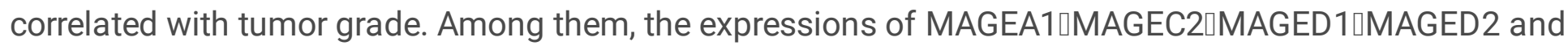
MAGEF1 were increased with tumor grade, especially for of MAGED1 and MAGEF1. Another MAGE gene, MAGEL2, was decreased with tumor grade.

Taken together, the results above suggested that the expression of 6 MAGE genes were significantly correlated with HCC patients' clinical stage and tumor grade .

\section{Prognostic value of MAGE genes in HCC}

Further, we analyzed the prognostic value of distinct MAGE genes in HCC using KM-Plot (http://kmplot.com/analysis/). As shown in fig.3, higher expressions of MAGEA6 ( $\mathrm{HR}=1.89,95 \% \mathrm{Cl}$ : 1.32 2.71, and $p=0.00043$ ) $\square$ MAGEA12 (HR=1.89, 95\% Cl: 1.31-2.71, and $p=0.00051)$ DMAGED1 ( HR=1.61, 95\% Cl: 1.03-2.51, and $p=0.034) \square M A G E D 2(H R=1.57,95 \% \mathrm{Cl}: 1.09-2.27$, and $p=0.015)$ were associated with worse prognosis of HCC. Higher expressions of MAGEA10 (HR=0.44, 95\% Cl: 031-0.62, and $p=1.6 \mathrm{e}-06) \square$ MAGEB4 (HR=0.35, 95\% Cl: 0.25-0.49, and $p=6.3 e-10)$ MAGEC3 (HR=0.5, 95\% Cl: 0.35-0.71, and $p=8.6 e-$ 05) $\triangle$ MAGEL2 (HR=0.67, 95\% Cl: $0.47-0.96$, and $p=0.029)$ were associated with better prognosis of HCC. Other members of MAGE family had no significant correlation with HCC prognosis. These results

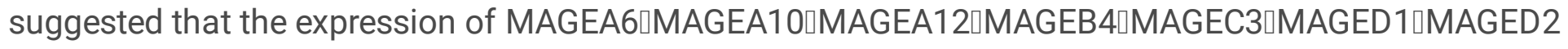
and MAGEL2 may serve as useful biomarkers for predicting HCC prognosis. 


\section{Diagnostic value of MAGE genes in HCC}

Based on the above results, we wandered to further evaluate the diagnostic value of distinct MAGE genes in HCC by computing receiver operating characteristic (ROC) curves. As shown in table 1 , the levels of MAGED1 (Area $=0.766,95 \%$ Cl: 0.719-0.813, and P<0.0001) and MAGED2 (Area=0.864, 95\% Cl: 0.8290.899 , and $P<0.0001$ ) were correlated with HCC incidence. Detailed ROC curves were shown in fig.4. These results suggest that the expressions of MAGED1 and MAGED2 may be exploited as useful biomarkers to diagnose HCC.

\section{Genetic alterations in MAGE genes and their associations with OS of HCC patients}

To further comprehensive evaluate the roles of dysregulated MAGEs in HCC, we analyzed genetic alteration in MAGE genes and their associations with OS of HCC patients in cBioPortal (https://www.cbioportal.org/). As shown in fig.5A, multiple MAGE genes had high genetic alteration rate in HCC patients. Among them, MAGEF1LMAGEA6IMAGEA1 and MAGEA12 ranked the highest four genes with genetic alterations, and their alteration rates were $12 \%, 12 \%, 11 \%$ and $10 \%$. Then, we chose the MAGEs with alteration rate over $8 \%$ for further OS analysis in $372 \mathrm{HCC}$ patients. As shown in fig.5B, the genetic alterations of MAGEs were not enough to distinguish HCC patients' prognosis. But the patients

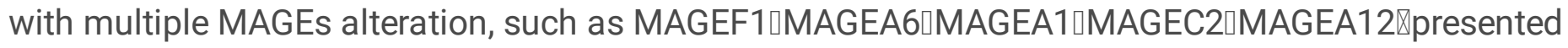
relatively worse prognosis, especially with genetic alterations of MAGEF1 and MAGEC2, although with not statistical difference. The results above suggested more attention is required to focus on the genetic alterations of MAGEs which may affect HCC patients' prognosis and can be exploited as useful biomarkers to diagnosis and treatment HCC.

\section{Evaluate the expression and clinical relevance of MAGE genes in HCC tissues of our center}

The above analysis results suggests that multiple MAGE genes were dysregulated in HCC and significantly associated with the clinicopathological parameters of HCC patients. Then, we further confirmed the expression profile and clinical relevance of some MAGEs which had never been reported before in our HCC clinical samples. 23 HCC patients were enrolled in our study with paired tumor tissue and adjacent non-tumor tissue and detailed clinical information. The few previous studies on the function of MAGEs in HCC have mainly focused on MAGE-A members and MAGE-D members. Based on this research status and above analysis results, we selected MAGEL2, MAGEB4, MAGEC3 and MAGEF1 for further research. The q-RT PCR experiment was performed to detect the expression of the above MAGEs in 23 pairs of HCC tumor tissues and adjacent non-tumor tissues. As shown in fig.6, the expression of MAGEL2 (fig.6A), MAGEB4(fig.6C) and MAGEC3(fig.6D) were significantly down-regulated in HCC tissues and MAGEF1 was significantly up-regulated in HCC tissues which were consistent with the results obtained by the previous bioinformatics analysis. Moreover, we observed a very interesting phenomenon that the expression patterns of MAGEL2, MAGEB4 and MAGEC3 in HCC patients were very similar, which may be related to their belonging to the MAGE family and it is worth further research. 
Then, we further analyzed the correlation between the expression of above MAGEs and the clinicopathologic characteristics of HCC patients. Based on the qRT-PCR result of related MAGEs, the cohort of $23 \mathrm{HCC}$ patients was divided into low and high groups using median as the cut off. Chi-square test was used to analyze the relationship between MAGEs expression and multiple clinicopathological characteristics in HCC patients. As shown in table 2-5, the expressions of MAGEL2 (table 2), MAGEB4(table 4) and MAGEC3 (table 5)were significantly correlated with vascular invasion and AFP level of HCC patients and the expression of MAGEF1 was significantly associated with tumor size and tumor number of HCC patients. Taken together, the results obtained with the HCC samples of our center further proved that multiple MAGEs were dysregulated in $\mathrm{HCC}$ and correlated with clinicopathologic characteristics of HCC patients. In addition, MAGEL2, MAGEB4, MAGEC3 and MAGEF1 deserve further study.

\section{Functional enrichment analysis of MAGE genes in HCC}

To reveal the molecular mechanisms of MAGEs functioned in HCC, we calculated GSVA enrichment scores using GSVA algorithm and further identified the significantly correlated genes with GSVA scores in GSE14520 microarray data. Then, we used the correlated genes predicted the Molecular function, Biological process, Cellular component and Reactome pathway of the dysregulated MAGEs in HCC (fig.7). The Molecular function Circos map (fig.7A) showed that the dysregulated MAGE genes were closely related to ligand-gated ion channel activity. The Biological process Circos map (fig.7B) showed that the dysregulated MAGE genes were closely related to cell cycle process and developmental process. The Cellular component Circos map (fig.7C) showed that the dysregulated MAGE genes were closely related to transmembrane transporter complex and ion channel complex. The Reactome pathway Circos map (fig.7D) showed that the dysregulated MAGE genes were closely related to $G$ protein-coupled receptors (GPCRs) ligand binding, peptide ligand-binding receptor and $G$ alpha signaling events. Taken together, the above functional enrichment analysis suggested the dysregulated MAGEs may play important roles in signal transduction.

\section{Screening of core genes significantly correlated to the function of MAGE genes in HCC}

Based on correlated genes with MAGEs in HCC, we further draw the protein-protein interaction networks using STRING. As shown in fig.8A, it was a large and complex network with close interaction of proteins in some regions. Then, we isolated three major protein-protein interaction regions (fig8B-8D). As shown in fig.8B, there were 35 members in network 1 , most of them were related with GPCR ligand binding and signal transduction $[27,28]$. Among them, five receptor proteins, HRH4ロGALR1[NMUR1ロGRM8 and GPR37L1, were colored with red, which had the most frequent interactions with other proteins and might play pivotal roles in network 1. In fig.8C, there were 19 members in network 2, most of them were related with ubiquitin-specific protease activity and regulated cellular protein metabolism [29,30]. All of the five pivotal proteins, RNF14 FBBX4DFBX022DTRIM36 and UBE2G2, were ubiquitin ligase. In fig.8D, there were 17 members in network 3, most of them were also related with G-protein coupled activity and the

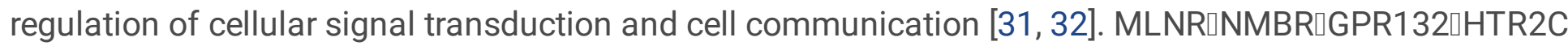


and LTB4R2, the five pivotal proteins in network 3, were all protein receptors related with G-protein. Taken together, the data above further suggested that the dysregulated MAGE genes were involved in the regulation of multiple pivotal intracellular signaling network. They might play important roles in the development of HCC and can be exploited as useful biomarkers to diagnosis and treatment HCC.

\section{Materials And Methods}

\section{Data sources}

GSE14520, a microarray data of human HCC which contains 225 tumor tissues and 220 non-tumor tissues, was download from NCBI GEO DataSets (https://www.ncbi.nlm.nih.gov/gds/?term=). The TCGA data was obtained through Genomic Data Commons Data Portal (https://portal.gdc.cancer.gov/). The platform contained $371 \mathrm{HCC}$ tissues and 50 adjacent non-tumor liver tissues. The data from the TCGA are publicly available and open access, and this research follows the TCGA data access policy and published guidelines.

\section{HCC specimens}

A total of 23 clinical HCC specimens used in this study were histopathologically and clinically diagnosed at the third affiliated hospital, Sun Yat-Sen University (Guangzhou, Guangdong, China). All samples were collected from 2017 to 2018 and each HCC tissue has paired non-tumorous tissues. For the use of these tissue in our study, we obtained approval from the Institutional Research Ethics Committee and informed written consent from all participants.

\section{Total RNA extraction and qRT-PCR}

Total RNA was isolated from tissue specimens using Trizol reagent (Invitrogen, Life Technologies Inc., Germany) according to the manufacturer's instructions. The density and purity of RNA were measured with NanoDrop 2000 (Thermo Fisher Scientific, USA). cDNA was synthesized by reverse transcription by First Strand cDNA Synthesis Kit for RT-PCR (Roche, Switzerland) according to the manufacturer's instructions. qRT-PCR was performed on LC480 real-time PCR detection system (Bio-Rad, Hercules, CA, USA), and a Roche SYBR FAST Universal qPCR Kit (Roche, Switzerland) was used for gene detection. All primers used for qRT-PCR were designed in primerBank (https://pga.mgh.harvard.edu/primerbank/index.html) and synthesized by BGITech (Beijing, China). GAPDH was used as the internal control. The sequences of primers are presented in Table S1.

\section{KM-Plot}

KM-Plot (http://kmplot.com/analysis/) is capable to evaluate the effect of 54k genes on survival in 21 cancer types. The datasets includes gene chip and RNA-seq data - sources for the databases include TCGA , GEO, and EGA. The main purpose of this tool is a meta-analysis based discovery and validation of survival biomarkers. In this research, we analyzed the correlation between MAGEs expression and the OS of HCC patients in KM-Plot. P values $<0.05$ were considered statistically significant. 


\section{UALCAN}

UALCAN (http://ualcan.path.uab.edu/index.html) is a comprehensive, user-friendly, and interactive web resource for analyzing cancer OMICS data. UALCAN is designed to, a) provide easy access to publicly available cancer OMICS data (TCGA and MET500), b) allow users to identify biomarkers or to perform in silico validation of potential genes of interest, c) provide graphs and plots depicting gene expression and patient survival information based on gene expression, d) evaluate gene expression in molecular subtypes of breast and prostate cancer, e) evaluate epigenetic regulation of gene expression by promoter methylation and correlate with gene expression, $\mathrm{f}$ ) perform pan-cancer gene expression analysis, and $\mathrm{g}$ ) Provide additional information about the selected genes/targets by linking to HPRD, GeneCards, Pubmed, TargetScan, The human protein atlas, DRUGBANK, Open Targets and the GTEx. These resources allow researchers to gather valuable information and data about the genes/targets of interest[33]. In this research, we analyzed the expression of MAGEs between HCC and non-tumor tissues and the association between MAGEs expression and clinicopathological characteristics of HCC patients, including clinical stage and differentiation. $P$ values $<0.05$ were considered statistically significant.

\section{Diagnostic prediction}

In this research, we plotted the ROC curves in SPSS based on the expression of MAGEs in HCC tissues and non-tumor tissues. The ROC curves were used to evaluate the predictive power of the dysregulated MAGEs in HCC diagnosis. The area under the curve $>0.7$ was considered with diagnostic value.

\section{cBioPortal}

cBioPortal (https://www.cbioportal.org) provides a Web resource for exploring, visualizing, and analyzing multidimensional cancer genomics data. The portal reduces molecular profiling data from cancer tissues and cell lines into readily understandable genetic, epigenetic, gene expression, and proteomic events. The query interface combined with customized data storage enables researchers to interactively explore genetic alterations across samples, genes, and pathways and, when available in the underlying data, to link these to clinical outcomes[34] . In this study, we analyzed the genomic profiles of 16 MAGE genes, including mutations, putative copy-number alterations from GISTIC and mRNA Expression z-Scores (RNASeq V2 RSEM) with a z-score threshold \pm 1.8 . The MAGE genes which genetic alteration rate exceeded $8 \%$ were further evaluated the correlation between the genetic alteration and the OS of HCC patients. $P$ values $<0.05$ were considered statistically significant.

\section{GSVA algorithm}

The GSVA package implements a non-parametric unsupervised method, called Gene Set Variation Analysis (GSVA), for assessing gene set enrichment (GSE) in gene expression microarray and RNA-seq data [35]. GSVA provides increased power to detect subtle pathway activity changes over a sample population in comparison to corresponding methods. While GSE methods are generally regarded as end points of a bioinformatic analysis, GSVA constitutes a starting point to build pathway-centric models of 
biology. Moreover, GSVA contributes to the current need of GSE methods for RNA-seq data. GSVA is an open source software package for $\mathrm{R}$ which forms part of the Bioconductor project and can be downloaded at http://www.bioconductor.org. In this research, we identified the significantly correlated genes with MAGEs in GSE14520 microarray data using GSVA algorithm. The $|r|>0.3$ was considered with significant correlation.

\section{Functional enrichment analysis}

Based on the significantly correlated genes with MAGEs, the functional enrichment analysis were conducted using clusterProfiler, biomaRt, GOplot and ReactomePA packages in R. The results were shown in the form of four circos plots which were generated using the Circos visualization tool in R [36]. In the circos plot, significantly correlated genes were presented in left and the related gene ontology (GO) terms were presented in right which different colors represented different $\mathrm{GO}$ terms and genes are connected by lines to the corresponding $\mathrm{GO}$ terms. $\mathrm{GO}$ terms with $\mathrm{P}<0.05$ were considered statistically significant.

\section{Protein-protein interaction network analysis}

Based on the significantly correlated genes with MAGEs, the protein-protein interaction network analysis was conducted using STRING (https://string-db.org/). Then, we further isolated the major protein-protein interaction regions using STRING results in Cytoscape version 3.3.1

(http://www.cytoscape.org/cy3.html). The genes with red color represented more frequent interactions with other proteins in networks.

\section{Statistical methods}

LIMMA Package in R was used to analyze the MAGEs expression in HCC tissues compared to non-tumor tissues of GSE14520, and differences in transcriptional expression was conducted using Student's t-test. $P$ values $<0.05$ were considered statistically significant. The ROC curves were plotted using the SPSS software version 22.0. The area under the curve $>0.7$ was considered with diagnostic value.

\section{Discussion}

HCC, accounting for $75 \%-85 \%$ of liver cancer, is a highly malignant carcinoma with high recurrence and chemoresistance all over the world[1]. Only a few treatments are available for HCC patients. Among them, surgical resection is still the most effective therapy to HCC patients. Even with surgical resection, the fiveyear recurrence is also as high as 70\% [2,3]. Moreover, most HCC patients lose the chance of surgical resection due to untimely diagnosis. The incurable and high recurrence of HCC are significantly correlated with the extremely complicated pathogenesis of HCC which multiple molecules and signal pathways have participated [37, 38]. Although with the continuous development of science and technology in recent years, researchers have clarified lots of molecules and signaling pathways related to HCC, the treatment of HCC has not been significantly improved. Further searching for molecules significantly correlated to the occurrence and development of $\mathrm{HCC}$ and revealing their functions are still urgent tasks for the 
majority of researchers, which will provide more effective targets for the diagnosis and treatment of HCC. In this research, we integrated and analyzed nearly 600 HCC patients' data from NCBI GEO DataSets and TCGA with authoritative bioinformatics websites and algorithms to reveal that multiple MAGE genes were dysregulated in HCC and significantly correlated with HCC clinicopathological characteristics, including clinical stage, tumor grade and OS. We further confirmed the expression and clinical relevance of MAGE genes in HCC tissues of our center, which suggested the MAGE genes are suitable to serve as new targets to diagnosis and treatment HCC.

The MAGE gene first attracted widespread attentions from oncologists due to its strong tumor antigen properties in melanoma. Subsequent series of studies further show that the dysregulation of MAGE genes exist in a variety of tumors, such as lung cancer, colon cancer, glioma, breast cancer, and prostate cancer[11-24]. The dysregulated MAGEs in cancers are often associated with worse clinical prognosis of tumor patients. However, the function of distinct MAGE genes in HCC is largely unclear. Kouichirou Tahara[26] and his colleagues first detected the expression of MAGEA1-A12 in 22 HCC tissues using qRTPCR, immunoblotting and immunohistochemistry. Their research suggested that multiple MAGEs were positively expressed in tumor tissues compared to non-tumor tissues. However, because the sample size is too small, the correlation between MAGEs expression and clinicopathological characteristics needs to be further verified. Soon after D-C Mou [39] tested the expression of MAGEA1 and MAGEA3 in the peripheral blood of $30 \mathrm{HCC}$ patients by nested RT-PCR and found that detection of MAGE transcripts with follow-up survey in PBMC is a feasible and reliable assay for the early prediction of the relapse and prognosis of the HCC patients. Zhao H [40] examined the expression of MAGEA4 in HCC and analyzed the relationship between positive expression rate of MAGEA4 and other clinical and lab data including AFP, AFU, anti-HCV, HBsAg, AFP mRNA, and the diameter of the tumors. Their research suggested that MAGEA4 might be served as a target for immunotherapy in HCC patients, but the expression of MAGEA4 has no correlation with HCC metastasis and recurrence. Hideki Takami [41] evaluated the expression of MAGED4 in Japanese HCC patients and found that overexpression of MAGED4 may be a predictive marker of early recurrence and mortality in patients with HCC. Hashimoto R and his colleagues [42, 43] analyzed the expression of MAGED1 and MAGED2 in nine HCC cell lines and 151 pairs of surgical tissues and indicated that MAGED1 and MAGED2 affect tumor progression and may serve as novel biomarkers and molecular targets for the treatment of HCC. Xuefeng Gu [44] detected the expression of MAGEA9 in HCC and analyzed the association between MAGEA9 expression and the clinicopathological characteristics of HCC. The results indicated that MAGEA9 expression is a valuable prognostic biomarker for HCC and high MAGEA9 expression suggests unfavorable survival outcomes in HCC patients. Subsequently, Youping Wei [45] found that high expression of MAGEA9 contributes to stemness and malignancy of HCC.

The MAGE family contains nearly 60 members, including type I MAGEs (MAGE-A, MAGE-B and MAGE-C subfamily members) and type II MAGEs (MAGE-D, MAGE-E, MAGE-F, MAGE-G, MAGE-H, MAGE-L and Necdin). However, previous studies on MAGEs in HCC have mainly focused on the MAGE-A superfamily and the HCC samples involved in previous studies are not enough. Based on these issues, we integrated and analyzed almost 600 HCC patients' data from NCBI GEO DataSets and TCGA using KM-Plot, 
UALCAN, cBioPortal databases and GSVA algorithm to comprehensive evaluate the function and clinical significance of MAGE genes in HCC. Our study revealed that multiple MAGE genes were dysregulated in

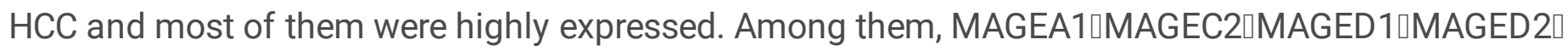
MAGEF1 and MAGEL2 were significantly correlated with HCC patients' clinical stage and tumor grade.

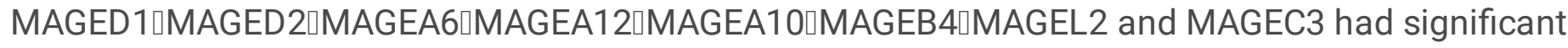
correlation with HCC prognosis. We also analyzed the functions of MAGEA3, MAGEA4, MAGEA9 and MAGED4 using data from NCBI GEO DataSets and TCGA, which had been reported in previous studies. Consistent with previous reports, MAGEA3 was up-regulated in $\mathrm{HCC}$ and correlated with shorter OS of HCC patients (data not shown). However, the expression of MAGEA3 was very low, which were also seen in MAGEA4, MAGEA9 and MAGED4 (data not shown), so we did not doing further research on them. Other unmentioned MAGE proteins were not analyzed due to low expression or undetected in HCC.

Hashimoto R and his colleagues [42, 43] had analyzed the expression of MAGED1 and MAGED2 in HCC and indicated that MAGED1 and MAGED2 affect tumor progression and may serve as novel biomarkers and molecular targets for the treatment of HCC. In our research, we further evaluated the diagnostic value of MAGED1 and MAGED2 in HCC by computing ROC curves. The detailed ROC results suggest that MAGED1 (Area $=0.766,95 \% \mathrm{Cl}: 0.719-0.813$, and P<0.0001), and MAGED2 (Area $=0.864,95 \% \mathrm{Cl}$ : $0.829-0.899$, and $\mathrm{P}<0.0001$ ) were significantly correlated with $\mathrm{HCC}$ incidence and may be exploited as useful biomarkers to diagnose HCC.

Gene abnormal alteration, including amplification, deletion and mutation are often correlated with the development of cancers [46]. We further analyzed genetic alteration in MAGE genes and their associations with OS of HCC patients. Analysis results showed that multiple MAGE genes had high genetic alteration rate in $\mathrm{HCC}$ and the patients with MAGE genetic alteration presented relatively worse prognosis, although with not statistical difference. More attention should be required to focus on the genetic alterations of MAGE genes in HCC which can be exploited as useful biomarkers to diagnosis and treatment HCC.

Previous studies on MAGEs in HCC mainly analyzed the expression of MAGEs in HCC and their association with the clinicopathological characteristics. However, it is largely unclear how dysregulated MAGE genes function in HCC. Recent biochemical and biophysical studies indicate that MAGEs assemble with E3 RING ubiquitin ligases to form MAGE-RING ligases (MRLs) and act as regulators of ubiquitination by modulating ligase activity, substrate specification, and subcellular localization [5]. Do the MAGE genes functioned in $\mathrm{HCC}$ depending on their regulation of the ubiquitin proteasome pathway? To clarify the molecular mechanisms of MAGE genes functioned in HCC, we analyzed the correlated genes of MAGEs in HCC using GSVA algorithm and further predicted the related Molecular function, Biological process, Cellular component and Reactome pathway. Based on the correlated genes of MAGEs, we also draw the protein-protein interaction networks using STRING. 15 core proteins, including HRH4ロGALR1DNMUR1D

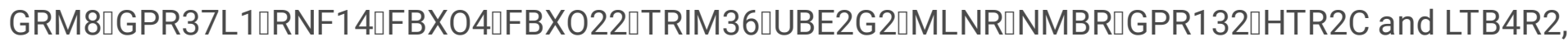
were found to be significantly correlated to the function of MAGEs in HCC. Most of them were G-protein receptors and ubiquitin ligase, involved in regulating GPCR ligand binding, ubiquitin-specific protease 
activity, cellular protein metabolism and signal transduction, which suggested the dysregulated MAGEs may play important roles in signal transduction of HCC cells and part of MAGEs function depend on their regulation of the ubiquitin proteasome pathway.

There are also limitations in our research. We analyzed the expression of MAGEs in HCC and the correlation between MAGEs expression and HCC clinicopathological characteristics using MAGEs mRNA levels in NCBI GEO DataSets and TCGA. Then, we verified the expression and clinical relevance of MAGE genes in HCC tissues of our center $(\mathrm{N}=23)$. More studies are needed to evaluate the functions of MAGEs in HCC using protein levels in larger HCC samples with the protein level of MAGEs. Moreover, the molecular mechanisms underlying MAGEs functioned in HCC need to be further investigated through comprehensive in vivo and vitro experiments and we will keep focusing on this issue in our future studies.

\section{Conclusions}

our research revealed that multiple MAGE genes were dysregulated in $\mathrm{HCC}$ and significant correlation with HCC prognosis. The dysregulated MAGEs were involved in the regulation of multiple signal transduction, might play important roles in the development of HCC and can be exploited as useful biomarkers to diagnosis and treatment HCC.

\section{Supplementary information}

Additional file 1: Table S1. The list of qRT-PCR primers used in this research

\section{Abbreviations}

$\mathrm{HCC}$

hepatocellular carcinoma; MAGE:Melanoma Antigen Gene; TCGA:The Cancer Genome Atlas; KMPlot:Kaplan-Meier Plotter; GSVA:Gene Expression Profiling Interactive Analysis; OS:overall survival; GPCR:G protein-coupled receptors; GO:gene ontology.

\section{Declarations}

\section{Ethics approval and consent to participate}

Our research was approved by the Ethics Committee of the Third Affiliated Hospital of Sun Yat-sen University. All HCC data involved in this research were retrieved from the online databases (NCBI GEO DataSets and TCGA) which could be confirmed that all written informed consent had already been obtained and the data acquisition were followed the related data access policy and published guidelines.

\section{Consent for publication}

Not applicable.

\section{Availability of data and materials}


The datasets used during the current study are available from the corresponding author on reasonable request.

Competing interests

The authors have no conflicts of interest to declare.

Funding Sources

This project was supported by: the National Natural Science Foundation of China, 81802897,81773176; Natural Science Foundation of Guangdong Province, 2018A030313259, 2016A030313250, 2017A030313715; Science and Technology Program of Guangdong Province, 2017B030314027; Science and Technology Program of Guangzhou, 201707010006, 201804010474 and the Fundamental Research Funds for the Central Universities, $19 y k p y 18$.

Author Contributions

R.L, J.G, Y.J, W.L and Y.C: designed the research; R.L, J.G, C.X: carried out the research; S.Z, J.L, Z.H, X.L, X.Y, X.Z and D.L: analyzed the data; R.L, J.G, C.X: wrote the paper, Y.J, W.L and Y.C: review \& editing the manuscript.

Acknowledgement

Not applicable.

\section{References}

1. Bray F, Ferlay J, Soerjomataram I, Siegel RL, Torre LA, Jemal A. Global cancer statistics 2018 : GLOBOCAN estimates of incidence and mortality worldwide for 36 cancers in 185 countries. Cancer J Clin. 2018;68(6):394-424.

2. Marquardt JU, Andersen JB, Thorgeirsson SS. Functional and genetic deconstruction of the cellular origin in liver cancer. Nature reviews Cancer. 2015;15(11):653-67.

3. Franssen B, Alshebeeb K, Tabrizian P, Marti J, Pierobon ES, Lubezky N, Roayaie S, Florman S, Schwartz ME. Differences in surgical outcomes between hepatitis B- and hepatitis C-related hepatocellular carcinoma: a retrospective analysis of a single North American center. Annals of surgery. 2014;260(4):650-6. discussion 656-658.

4. Llovet JM, Schwartz M, Mazzaferro V. Resection and liver transplantation for hepatocellular carcinoma. Semin Liver Dis. 2005;25(2):181-200.

5. Lee AK, Potts PR. A Comprehensive Guide to the MAGE Family of Ubiquitin Ligases. Journal of molecular biology 2017, 429(8):1114-1142.

6. Chomez P, De Backer O, Bertrand M, De Plaen E, Boon T, Lucas S. An overview of the MAGE gene family with the identification of all human members of the family. Cancer research. 2001;61(14):5544-51.

7. Weon JL, Potts PR. The MAGE protein family and cancer. Curr Opin Cell Biol. 2015;37:1-8. 
8. Lian Y, Meng L, Ding P, Sang M. Epigenetic regulation of MAGE family in human cancer progressionDNA methylation, histone modification, and non-coding RNAs. Clinical epigenetics. 2018;10(1):115.

9. Barker PA, Salehi A. The MAGE proteins: emerging roles in cell cycle progression, apoptosis, and neurogenetic disease. J Neurosci Res. 2002;67(6):705-12.

10. De Donato M, Peters SO, Hussain T, Rodulfo H, Thomas BN, Babar ME, Imumorin IG. Molecular evolution of type II MAGE genes from ancestral MAGED2 gene and their phylogenetic resolution of basal mammalian clades. Mammalian genome: official journal of the International Mammalian Genome Society. 2017;28(9-10):443-54.

11. Wang D, Wang J, Ding N, Li Y, Yang Y, Fang X, Zhao H. MAGE-A1 promotes melanoma proliferation and migration through C-JUN activation. Biochem Biophys Res Commun. 2016;473(4):959-65.

12. Barrow C, Browning J, MacGregor D, Davis ID, Sturrock S, Jungbluth AA, Cebon J. Tumor antigen expression in melanoma varies according to antigen and stage. Clinical cancer research: an official journal of the American Association for Cancer Research. 2006;12(3 Pt 1):764-71.

13. Ravichandran R, Kodali K, Peng J, Potts PR. Regulation of MAGE-A3/6 by the CRL4-DCAF12 ubiquitin ligase and nutrient availability. EMBO Rep. 2019;20(7):e47352.

14. Vansteenkiste JF, Cho BC, Vanakesa T, De Pas T, Zielinski M, Kim MS, Jassem J, Yoshimura M, Dahabreh J, Nakayama H, et al. Efficacy of the MAGE-A3 cancer immunotherapeutic as adjuvant therapy in patients with resected MAGE-A3-positive non-small-cell lung cancer (MAGRIT): a randomised, double-blind, placebo-controlled, phase 3 trial. The Lancet Oncology. 2016;17(6):82235 .

15. Duperret EK, Liu S, Paik M, Trautz A, Stoltz R, Liu X, Ze K, Perales-Puchalt A, Reed C, Yan J, et al. A Designer Cross-reactive DNA Immunotherapeutic Vaccine that Targets Multiple MAGE-A Family Members Simultaneously for Cancer Therapy. Clinical cancer research: an official journal of the American Association for Cancer Research. 2018;24(23):6015-27.

16. Yang B, O'Herrin SM, Wu J, Reagan-Shaw S, Ma Y, Bhat KM, Gravekamp C, Setaluri V, Peters N, Hoffmann FM, et al: MAGE-A, mMage-b, and MAGE-C proteins form complexes with KAP1 and suppress p53-dependent apoptosis in MAGE-positive cell lines. Cancer research 2007, 67(20):99549962.

17. Takahashi N, Ohkuri T, Homma S, Ohtake J, Wakita D, Togashi Y, Kitamura H, Todo S, Nishimura T. First clinical trial of cancer vaccine therapy with artificially synthesized helper/ killer-hybrid epitope long peptide of MAGE-A4 cancer antigen. Cancer Sci. 2012;103(1):150-3.

18. Zeng ZL, Wu WJ, Yang J, Tang ZJ, Chen DL, Qiu MZ, Luo HY, Wang ZQ, Jin Y, Wang DS, et al. Prognostic relevance of melanoma antigen D1 expression in colorectal carcinoma. Journal of translational medicine. 2012;10:181.

19. Liu XL, Zhao D, Sun DP, Wang Y, Li Y, Qiu FQ, Ma P. Adenovirus-mediated delivery of CALR and MAGEA3 inhibits invasion and angiogenesis of glioblastoma cell line U87. Journal of experimental clinical cancer research: CR. 2012;31:8. 
20. Weeraratne SD, Amani V, Neiss A, Teider N, Scott DK, Pomeroy SL, Cho YJ. miR-34a confers chemosensitivity through modulation of MAGE-A and p53 in medulloblastoma. Neurooncology. 2011;13(2):165-75.

21. Zhao J, Wang Y, Mu C, Xu Y, Sang J. MAGEA1 interacts with FBXW7 and regulates ubiquitin ligasemediated turnover of NICD1 in breast and ovarian cancer cells. Oncogene. 2017;36(35):5023-34.

22. Raghavendra A, Kalita-de Croft P, Vargas AC, Smart CE, Simpson PT, Saunus JM, Lakhani SR. Expression of MAGE-A and NY-ESO-1 cancer/testis antigens is enriched in triple-negative invasive breast cancers. Histopathology. 2018;73(1):68-80.

23. Karpf AR, Bai S, James SR, Mohler JL, Wilson EM. Increased expression of androgen receptor coregulator MAGE-11 in prostate cancer by DNA hypomethylation and cyclic AMP. Molecular cancer research: MCR. 2009;7(4):523-35.

24. Kawada J, Wada H, Isobe M, Gnjatic S, Nishikawa H, Jungbluth AA, Okazaki N, Uenaka A, Nakamura $Y$, Fujiwara $S$, et al. Heteroclitic serological response in esophageal and prostate cancer patients after NY-ESO-1 protein vaccination. International journal of cancer. 2012;130(3):584-92.

25. Schooten E, Di Maggio A, van Bergen En Henegouwen PMP, Kijanka MM. MAGE-A antigens as targets for cancer immunotherapy. Cancer treatment reviews. 2018;67:54-62.

26. Tahara K, Mori M, Sadanaga N, Sakamoto Y, Kitano S, Makuuchi M. Expression of the MAGE gene family in human hepatocellular carcinoma. Cancer. 1999;85(6):1234-40.

27. Cai WK, Hu J, Li T, Meng JR, Ma X, Yin SJ, Zhao CH, He GH, Xu GL. Activation of histamine H4 receptors decreases epithelial-to-mesenchymal transition progress by inhibiting transforming growth factor-beta1 signalling pathway in non-small cell lung cancer. European journal of cancer. 2014;50(6):1195-206.

28. Wallrapp A, Riesenfeld SJ, Burkett PR, Abdulnour RE, Nyman J, Dionne D, Hofree M, Cuoco MS, Rodman C, Farouq D, et al. The neuropeptide NMU amplifies ILC2-driven allergic lung inflammation. Nature. 2017;549(7672):351-6.

29. Wu B, Piloto S, Zeng W, Hoverter NP, Schilling TF, Waterman ML. Ring Finger Protein 14 is a new regulator of TCF/beta-catenin-mediated transcription and colon cancer cell survival. EMBO Rep. 2013;14(4):347-55.

30. Liang C, Wang S, Qin C, Bao M, Cheng G, Liu B, Shao P, Lv Q, Song N, Hua L, et al. TRIM36, a novel androgen-responsive gene, enhances anti-androgen efficacy against prostate cancer by inhibiting MAPK/ERK signaling pathways. Cell death disease. 2018;9(2):155.

31. Pyo JS, Shin YM, Kang DW. Prognostic Implication of Metastatic Lymph Node Ratio in Colorectal Cancers: Comparison Depending on Tumor Location. Journal of clinical medicine 2019, 8(11).

32. Nii T, Prabhu VV, Ruvolo V, Madhukar N, Zhao R, Mu H, Heese L, Nishida Y, Kojima K, Garnett MJ, et al. Imipridone ONC212 activates orphan G protein-coupled receptor GPR132 and integrated stress response in acute myeloid leukemia. Leukemia. 2019;33(12):2805-16.

33. Chandrashekar DS, Bashel B, Balasubramanya SAH, Creighton CJ, Ponce-Rodriguez I, Chakravarthi B, Varambally S. UALCAN: A Portal for Facilitating Tumor Subgroup Gene Expression and Survival 
Analyses. Neoplasia. 2017;19(8):649-58.

34. Gao J, Aksoy BA, Dogrusoz U, Dresdner G, Gross B, Sumer SO, Sun Y, Jacobsen A, Sinha R, Larsson E, et al. Integrative analysis of complex cancer genomics and clinical profiles using the cBioPortal. Sci Signal. 2013;6(269):pl1.

35. Hanzelmann S, Castelo R, Guinney J. GSVA: gene set variation analysis for microarray and RNA-seq data. BMC Bioinform. 2013;14:7.

36. Chan EKF, Cameron DL, Petersen DC, Lyons RJ, Baldi BF, Papenfuss AT, Thomas DM, Hayes VM. Optical mapping reveals a higher level of genomic architecture of chained fusions in cancer. Genome research. 2018;28(5):726-38.

37. Llovet JM, Montal R, Sia D, Finn RS. Molecular therapies and precision medicine for hepatocellular carcinoma. Nature reviews Clinical oncology. 2018;15(10):599-616.

38. Ghouri YA, Mian I, Rowe JH. Review of hepatocellular carcinoma: Epidemiology, etiology, and carcinogenesis. Journal of carcinogenesis 2017, 16:1.

39. Mou DC, Cai SL, Peng JR, Wang Y, Chen HS, Pang XW, Leng XS, Chen WF. Evaluation of MAGE-1 and MAGE-3 as tumour-specific markers to detect blood dissemination of hepatocellular carcinoma cells. British journal of cancer. 2002;86(1):110-6.

40. Zhao H, Rui J, Cong X, Zhao Y, Wang S, Chen S, Wang Y, Wang Y, Leng X, Chen W. [Expression and clinical significance of MAGE-4 gene in human hepatocellular carcinoma]. Zhonghua gan zang bing za zhi = Zhonghua ganzangbing zazhi = Chinese journal of hepatology. 2002;10(2):100-2.

41. Takami H, Kanda M, Oya H, Hibino S, Sugimoto H, Suenaga M, Yamada S, Nishikawa Y, Asai M, Fujii T, et al. Evaluation of MAGE-D4 expression in hepatocellular carcinoma in Japanese patients. Journal of surgical oncology. 2013;108(8):557-62.

42. Hashimoto R, Kanda M, Takami H, Shimizu D, Oya H, Hibino S, Okamura Y, Yamada S, Fujii T, Nakayama G, et al. Aberrant expression of melanoma-associated antigen-D2 serves as a prognostic indicator of hepatocellular carcinoma outcome following curative hepatectomy. Oncology letters. 2015;9(3):1201-6.

43. Shimizu D, Kanda M, Sugimoto H, Sueoka S, Takami H, Ezaka K, Tanaka Y, Hashimoto R, Okamura Y, Iwata N, et al. NRAGE promotes the malignant phenotype of hepatocellular carcinoma. Oncology letters. 2016;11(3):1847-54.

44. Gu X, Fu M, Ge Z, Zhan F, Ding Y, Ni H, Zhang W, Zhu Y, Tang X, Xiong L, et al. High expression of MAGE-A9 correlates with unfavorable survival in hepatocellular carcinoma. Scientific reports. 2014;4:6625.

45. Wei Y, Wang Y, Gong J, Rao L, Wu Z, Nie T, Shi D, Zhang L. High expression of MAGE-A9 contributes to stemness and malignancy of human hepatocellular carcinoma. Int J Oncol. 2018;52(1):219-30.

46. Cancer Genome Atlas Research Network. Electronic address wbe, Cancer Genome Atlas Research N: Comprehensive and Integrative Genomic Characterization of Hepatocellular Carcinoma. Cell. 2017;169(7):1327-41. e1323. 


\section{Tables}

\begin{tabular}{|c|c|c|c|c|}
\hline \multirow[t]{2}{*}{ Gene } & \multirow[t]{2}{*}{ Area } & \multirow[t]{2}{*}{$P$ value } & \multicolumn{2}{|c|}{ 95\% Confidence Interval } \\
\hline & & & Lower Bound & Upper Bound \\
\hline MAGED1 & .766 & .000 & .719 & .813 \\
\hline MAGED2 & .864 & .000 & .829 & .899 \\
\hline MAGEF1 & .644 & .000 & .590 & .697 \\
\hline MAGEA6 & .666 & .000 & .616 & .717 \\
\hline MAGEA1 & .638 & .000 & .586 & .691 \\
\hline MAGEA12 & .656 & .000 & .605 & .707 \\
\hline MAGEC1 & .583 & .002 & .530 & .636 \\
\hline MAGEB2 & .614 & .000 & .562 & .666 \\
\hline MAGEA8 & .531 & .251 & .478 & .585 \\
\hline MAGEB1 & .543 & .114 & .490 & .597 \\
\hline MAGEA10 & .597 & .000 & .545 & .649 \\
\hline MAGEB4 & .662 & .000 & .612 & .712 \\
\hline MAGEL2 & .657 & .000 & .606 & .707 \\
\hline MAGEC2 & .673 & .000 & .623 & .722 \\
\hline MAGEC3 & .573 & .008 & .519 & .626 \\
\hline \multicolumn{5}{|c|}{$\begin{array}{l}\text { The test result variable(s): MAGED1, MAGED2, MAGEF1, MAGEA6, MAGEA1, MAGEA12, } \\
\text { MAGEC1, MAGEB2, MAGEA8, MAGEB1, MAGEA10, MAGEB4, MAGEL2, MAGEC2, MAGEC3 has at } \\
\text { least one tie between the positive actual state group and the negative actual state group. } \\
\text { Statistics may be biased. }\end{array}$} \\
\hline \multicolumn{5}{|c|}{ a. Under the nonparametric assumption } \\
\hline \multicolumn{5}{|c|}{ b. Null hypothesis: true area $=0.5$} \\
\hline
\end{tabular}




\begin{tabular}{|c|c|c|c|c|}
\hline \multirow[t]{2}{*}{ characteristics } & & \multicolumn{2}{|c|}{ MAGEL2 expression } & \multirow[t]{2}{*}{ Pearson's chi-square test ( $p$ value ${ }^{\star}$ ) } \\
\hline & & Low & High & \\
\hline \multirow[t]{2}{*}{ Gender } & Male & 10 & 7 & \multirow[t]{2}{*}{0.283} \\
\hline & Female & 2 & 4 & \\
\hline \multirow[t]{2}{*}{ Age } & $<60$ & 7 & 6 & \multirow[t]{2}{*}{0.855} \\
\hline & $\geqq 60$ & 5 & 5 & \\
\hline \multirow[t]{2}{*}{ Differentiation } & High & 8 & 9 & \multirow[t]{2}{*}{0.408} \\
\hline & Moderate/Low & 4 & 2 & \\
\hline \multirow[t]{2}{*}{ Tumor size } & $<5 \mathrm{~cm}$ & 5 & 2 & \multirow[t]{2}{*}{0.221} \\
\hline & $\geqq 5 \mathrm{~cm}$ & 7 & 9 & \\
\hline \multirow[t]{2}{*}{ Tumor number } & Single & 6 & 3 & \multirow[t]{2}{*}{0.265} \\
\hline & Multiple & 6 & 8 & \\
\hline \multirow[t]{2}{*}{ TNM stage } & $\mid+\|$ & 5 & 2 & \multirow[t]{2}{*}{0.221} \\
\hline & III+IV & 7 & 9 & \\
\hline \multirow[t]{2}{*}{ Vascular invasion } & No & 1 & 5 & \multirow[t]{2}{*}{0.043} \\
\hline & Yes & 11 & 6 & \\
\hline \multirow[t]{2}{*}{ AFP } & $<20$ & 1 & 6 & \multirow[t]{2}{*}{0.016} \\
\hline & $\geqq 20$ & 11 & 5 & \\
\hline
\end{tabular}




\begin{tabular}{|c|c|c|c|c|}
\hline \multirow[t]{2}{*}{ characteristics } & & \multicolumn{2}{|c|}{ MAGEF1 expression } & \multirow[t]{2}{*}{ Pearson's chi-square test ( $p$ value $\left.{ }^{\star}\right)$} \\
\hline & & Low & High & \\
\hline \multirow[t]{2}{*}{ Gender } & Male & 10 & 7 & \multirow[t]{2}{*}{0.283} \\
\hline & Female & 2 & 4 & \\
\hline \multirow[t]{2}{*}{ Age } & $<60$ & 6 & 7 & \multirow[t]{2}{*}{0.51} \\
\hline & $\geqq 60$ & 6 & 4 & \\
\hline \multirow[t]{2}{*}{ Differentiation } & High & 9 & 8 & \multirow[t]{2}{*}{0.901} \\
\hline & Moderate/Low & 3 & 3 & \\
\hline \multirow[t]{2}{*}{ Tumor size } & $<5 \mathrm{~cm}$ & 6 & 1 & \multirow[t]{2}{*}{0.033} \\
\hline & $\geqq 5 \mathrm{~cm}$ & 6 & 10 & \\
\hline \multirow[t]{2}{*}{ Tumor number } & Single & 7 & 2 & \multirow[t]{2}{*}{0.049} \\
\hline & Multiple & 5 & 9 & \\
\hline \multirow[t]{2}{*}{ TNM stage } & $1+\|$ & 4 & 3 & \multirow[t]{2}{*}{0.752} \\
\hline & III+IV & 8 & 8 & \\
\hline \multirow[t]{2}{*}{ Vascular invasion } & No & 2 & 4 & \multirow[t]{2}{*}{0.283} \\
\hline & Yes & 10 & 7 & \\
\hline \multirow[t]{2}{*}{ AFP } & $<20$ & 2 & 5 & \multirow[t]{2}{*}{0.134} \\
\hline & $\geqq 20$ & 10 & 6 & \\
\hline
\end{tabular}




\section{Table 4ロCorrelation between MAGEB4 expression level and the}

clinicopathologic characteristics of 23 cases of HCC patients

characteristics

MAGEB4 expression Pearson's chi-square test ( $p$ value*)

Low High

\begin{tabular}{lllll} 
Gender & Male & 10 & 7 & 0.283 \\
\cline { 2 - 5 } & Female & 2 & 4 & \\
\multirow{2}{*}{ Age } & $<60$ & 7 & 6 & 0.855 \\
& $\geqq 60$ & 5 & 5 &
\end{tabular}

$\begin{array}{lllll}\text { Differentiation } & \text { High } & 8 & 9 & 0.408\end{array}$

\begin{tabular}{lllll} 
& Moderate/Low & 4 & 2 & \\
\multirow{3}{*}{ Tumor size } & $<5 \mathrm{~cm}$ & 5 & 2 & 0.221 \\
& $\geqq 5 \mathrm{~cm}$ & 7 & 9 &
\end{tabular}

\begin{tabular}{|lllll|} 
Tumor number & Single & 6 & 3 & 0.265 \\
\cline { 2 - 4 } & Multiple & 6 & 8 & \\
\hline \multirow{2}{*}{ TNM stage } & I+II & 5 & 2 & 0.221 \\
\cline { 2 - 4 } & III+IV & 7 & 9 & \\
\hline Vascular invasion & No & 1 & 5 & 0.043 \\
\cline { 2 - 4 } & Yes & 11 & 6 & \\
\hline AFP & $<20$ & 1 & 6 & 0.016 \\
\cline { 2 - 4 } & $\geqq 20$ & 11 & 5 & \\
& & & &
\end{tabular}

*Results were considered statistically significant at $p<0.05$ 
Table 5ロCorrelation between MAGEC3 expression level and the clinicopathologic characteristics of 23 cases of HCC patients

\begin{tabular}{|c|c|c|c|c|}
\hline \multirow[t]{2}{*}{ characteristics } & & \multicolumn{2}{|c|}{ MAGEC3 expression } & \multirow[t]{2}{*}{ Pearson's chi-square test ( $p$ value*) } \\
\hline & & Low & High & \\
\hline \multirow[t]{2}{*}{ Gender } & Male & 10 & 7 & \multirow[t]{2}{*}{0.283} \\
\hline & Female & 2 & 4 & \\
\hline \multirow[t]{2}{*}{ Age } & $<60$ & 7 & 6 & \multirow[t]{2}{*}{0.855} \\
\hline & $\geqq 60$ & 5 & 5 & \\
\hline \multirow[t]{2}{*}{ Differentiation } & High & 8 & 9 & \multirow[t]{2}{*}{0.408} \\
\hline & Moderate/Low & 4 & 2 & \\
\hline \multirow[t]{2}{*}{ Tumor size } & $<5 \mathrm{~cm}$ & 5 & 2 & \multirow[t]{2}{*}{0.221} \\
\hline & $\geqq 5 \mathrm{~cm}$ & 7 & 9 & \\
\hline \multirow[t]{2}{*}{ Tumor number } & Single & 6 & 3 & \multirow[t]{2}{*}{0.265} \\
\hline & Multiple & 6 & 8 & \\
\hline \multirow[t]{2}{*}{ TNM stage } & $1+\|$ & 5 & 2 & \multirow[t]{2}{*}{0.221} \\
\hline & III+IV & 7 & 9 & \\
\hline \multirow[t]{2}{*}{ Vascular invasion } & No & 1 & 5 & \multirow[t]{2}{*}{0.043} \\
\hline & Yes & 11 & 6 & \\
\hline \multirow[t]{2}{*}{ AFP } & $<20$ & 1 & 6 & \multirow[t]{2}{*}{0.016} \\
\hline & $\geqq 20$ & 11 & 5 & \\
\hline
\end{tabular}

${ }^{*}$ Results were considered statistically significant at $p<0.05$

\section{Figures}



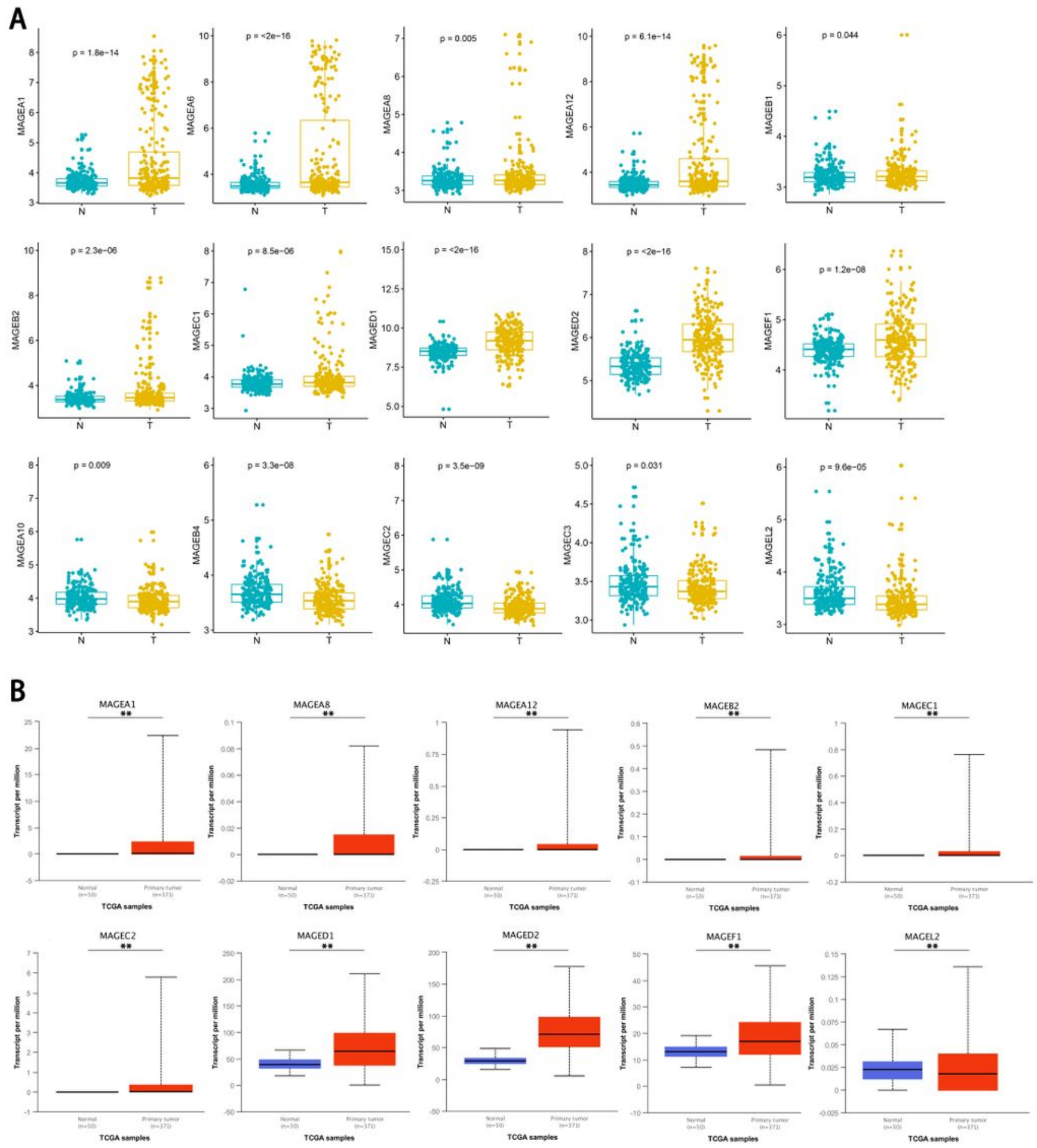

\section{Figure 1}

Multiple MAGE genes are dysregulated in HCC. (A-B) The mRNA expressions of MAGE genes in HCC tissues and non-tumor tissues (A: GSE14520, B: TCGA). Multiple MAGE genes were aberrantly expressed in HCC tissues and most of them were highly expressed. ${ }^{*} p<0.05,{ }^{\star \star} p<0.01,{ }^{\star \star \star} p<0.001$. 
A
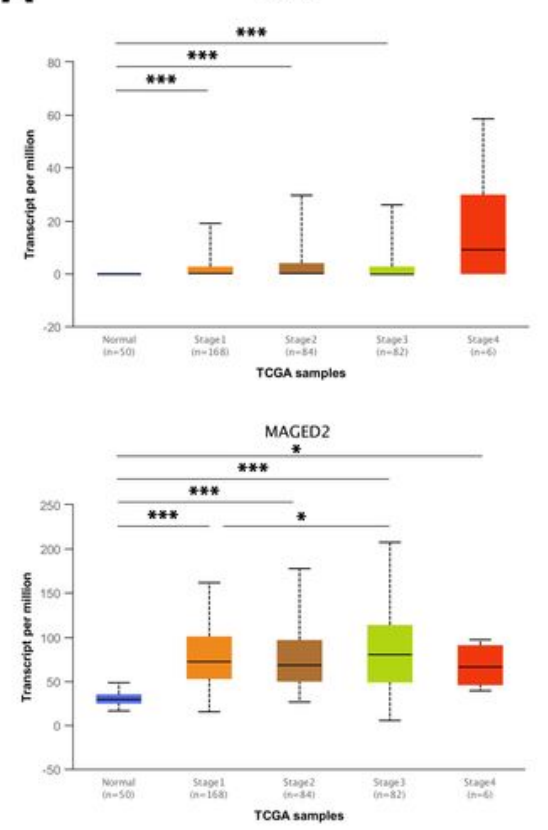

B
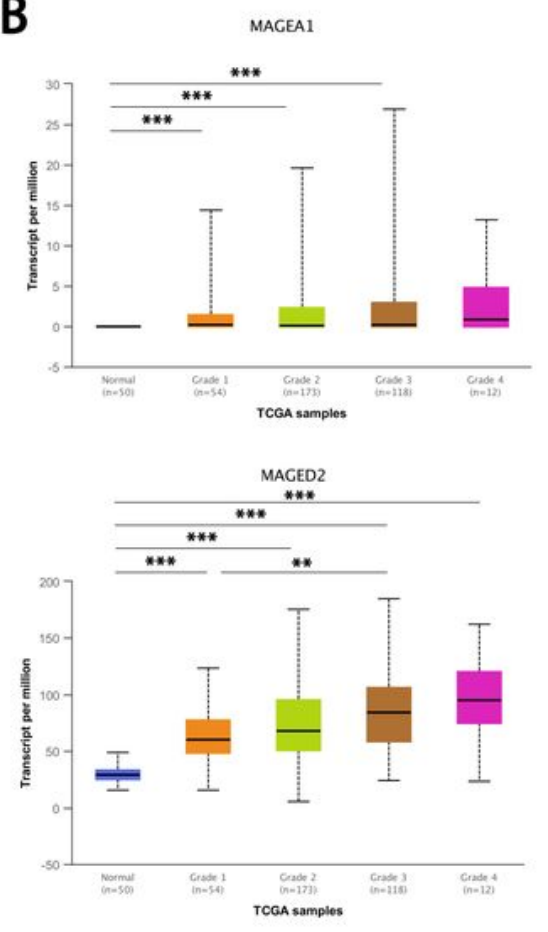
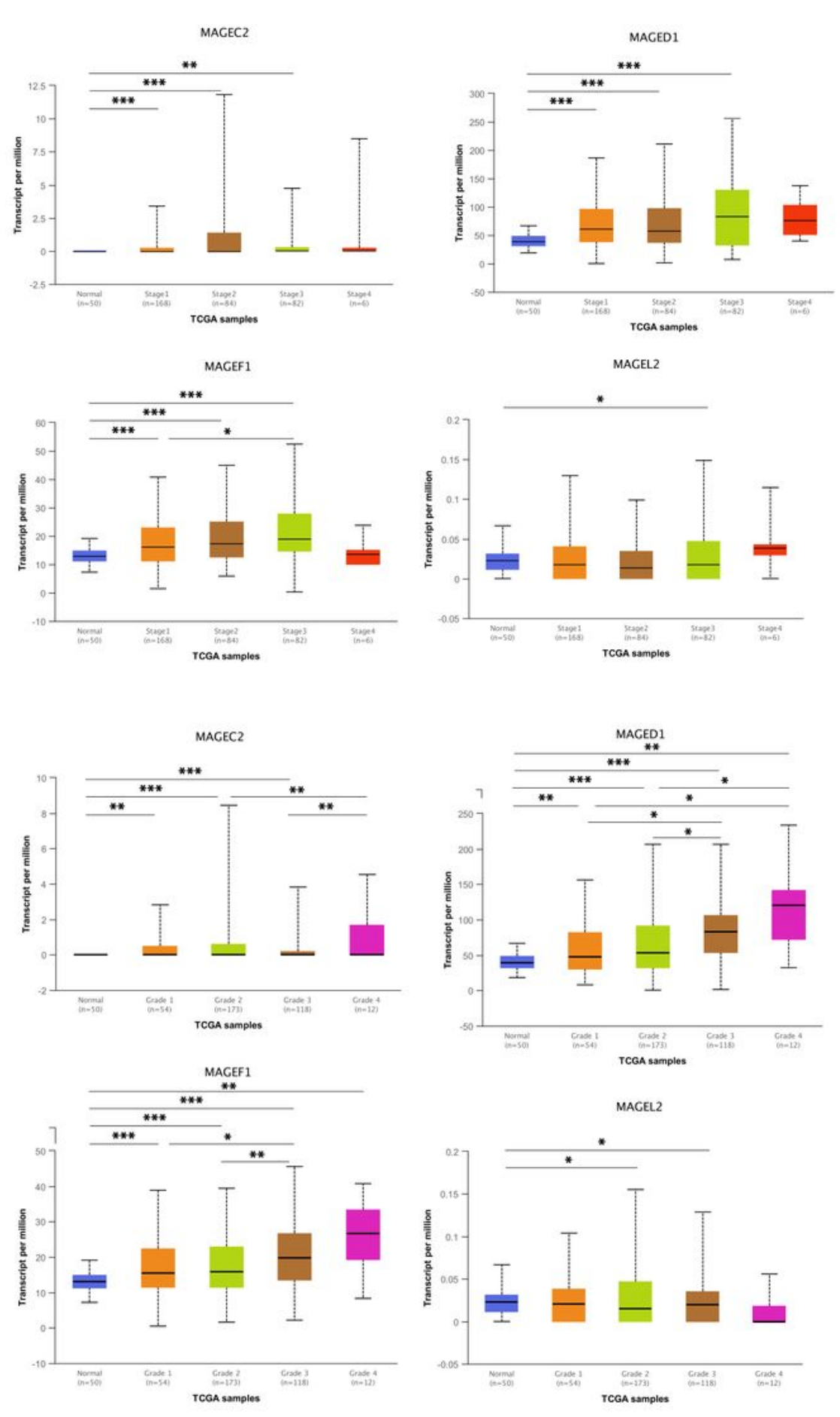

\section{Figure 2}

The correlation between the expression of dysregulated MAGE genes and clinicopathological characteristics of HCC patients. (A-B) mRNA expressions of 6 MAGE genes were significantly correlated with HCC patients' clinical stage and tumor grade. The expression of MAGEA1, MAGEC2, MAGED1, MAGED2, and MAGEF1 were increased with the clinical stage and tumor grade. Another MAGE family member, MAGEL2, was decreased with clinical stage and tumor grade . ${ }^{*} p<0.05,{ }^{* \star} p<0.01,{ }^{* \star *} p<0.001$. 

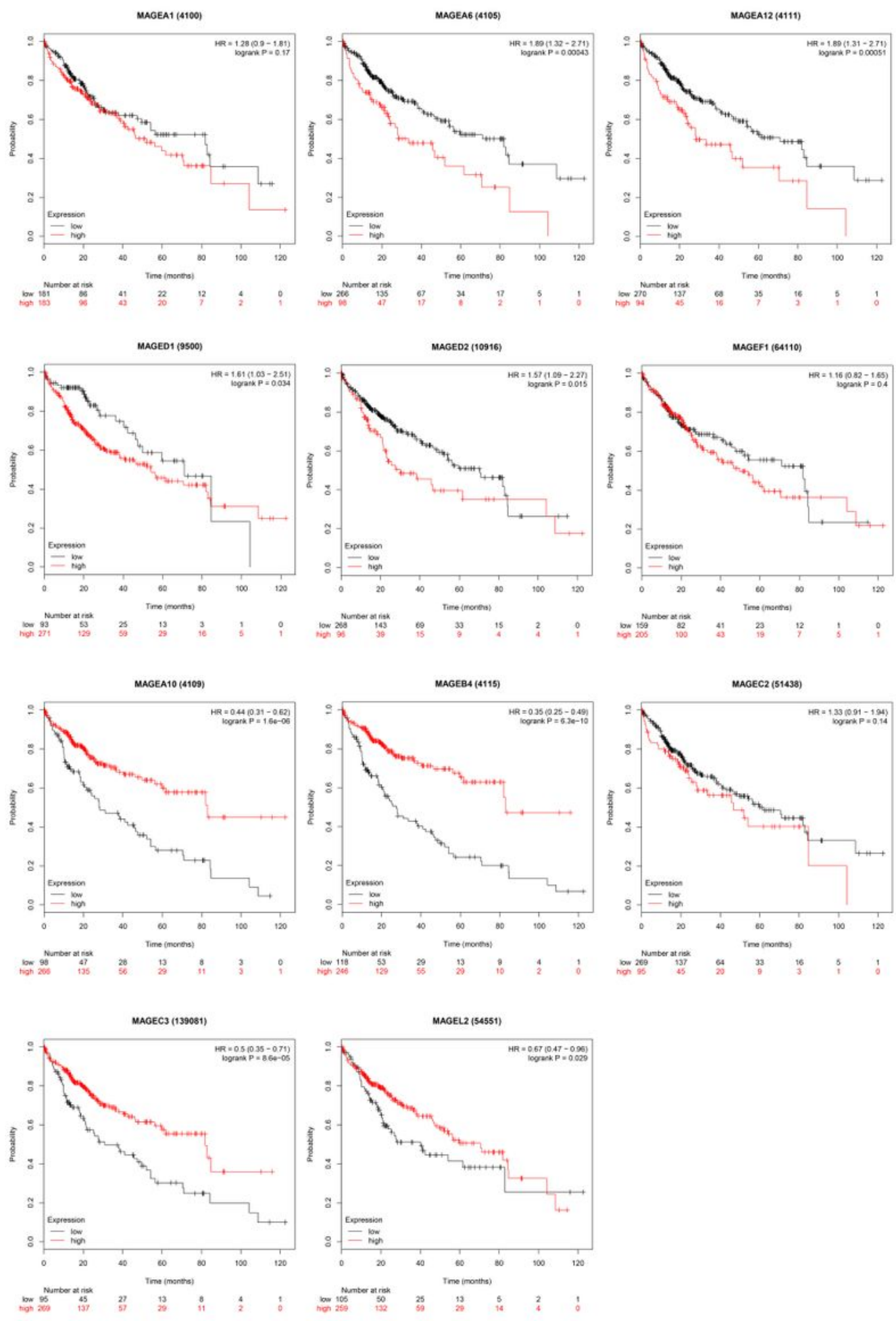

\section{Figure 3}

Prognostic value of the MAGEs mRNA expression in HCC patients. Higher expressions of MAGED1, MAGED2, MAGEA6 and MAGEA12 were associated with worse prognosis in HCC patients. Higher expressions of MAGEA10, MAGEB4, MAGEL2 and MAGEC3 were associated with better prognosis in HCC patients. The mRNA expression of other MAGE genes, including MAGEA1, MAGEF1 and MAGEC2, showed no correlation with HCC patients' prognosis. $\mathrm{P}$ values $<0.05$ were considered statistically significant. 

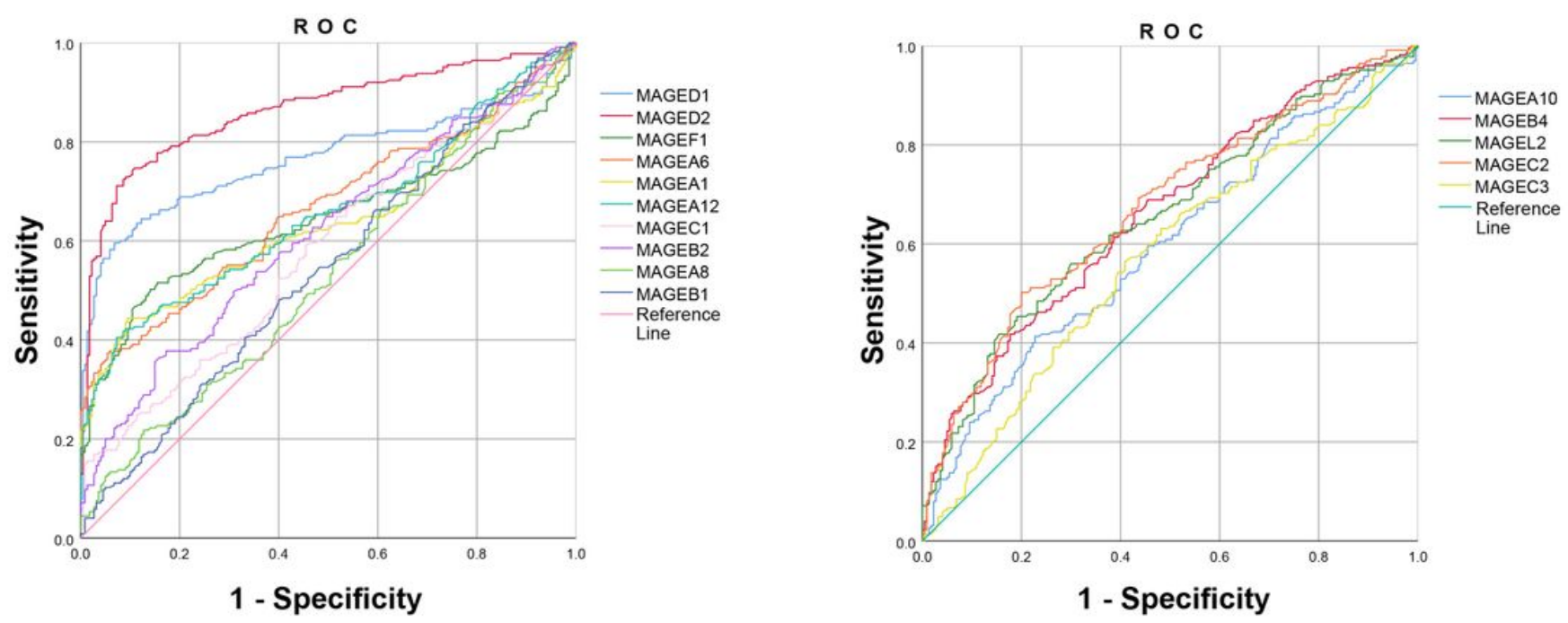

Figure 4

Diagnostic value of the MAGEs mRNA expression in HCC patients. According to the expression of MAGE genes in HCC tissues and adjacent non-tumor tissues, we divided all dysregulated MAGE genes into two groups, namely the high expression group and the low expression group. The ROC curves of the two groups were drawn separately, the ROC curve on the left represented the high expression group and the ROC curve on the right represented the low expression group. Among the 15 MAGEs, only MAGED1 and MAGED2 were considered correlated with HCC incidence. The area under the curve $>0.7$ was considered with diagnostic value. 
A
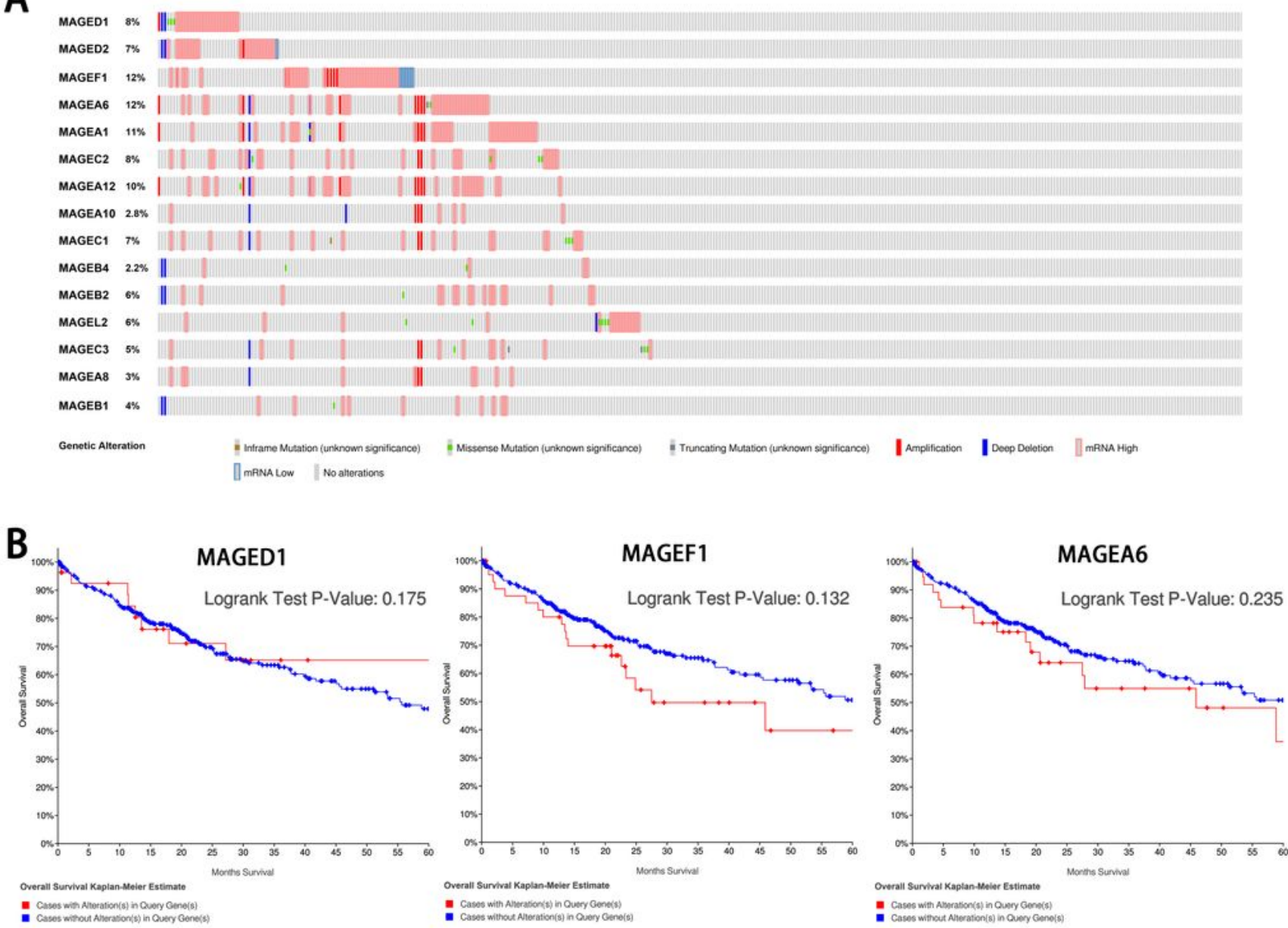

- Casos with Aloration(s) in Cunty Oeno(s)

Overall Survirat Kaplan Mover Estimato

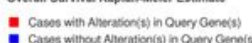

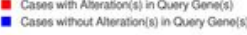
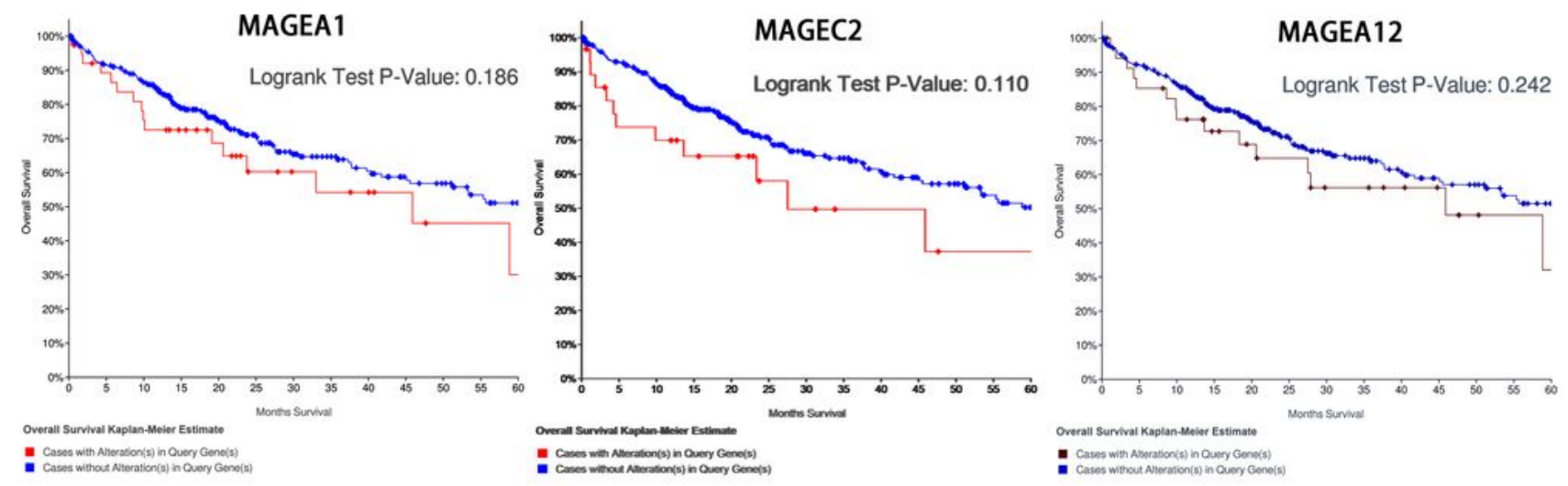

\section{Figure 5}

Genetic alterations in MAGEs and their correlation with OS of HCC patients. (A) Multiple MAGE genes had high genetic alteration rate in HCC patients. Among them, MAGEF1DMAGEA6ロMAGEA1 and MAGEA12 ranked the highest four genes with genetic alterations, and their alteration rates were $12 \%, 12 \%, 11 \%$ and $10 \%$. (B) We chose the MAGE genes with alteration rate over $8 \%$ for further OS analysis. Patients with

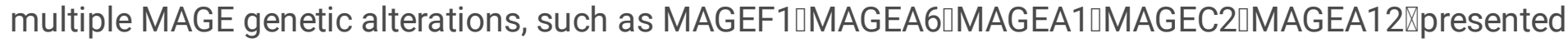


relatively worse prognosis, although with not statistical difference. $\mathrm{P}$ values $<0.05$ were considered statistically significant.

A

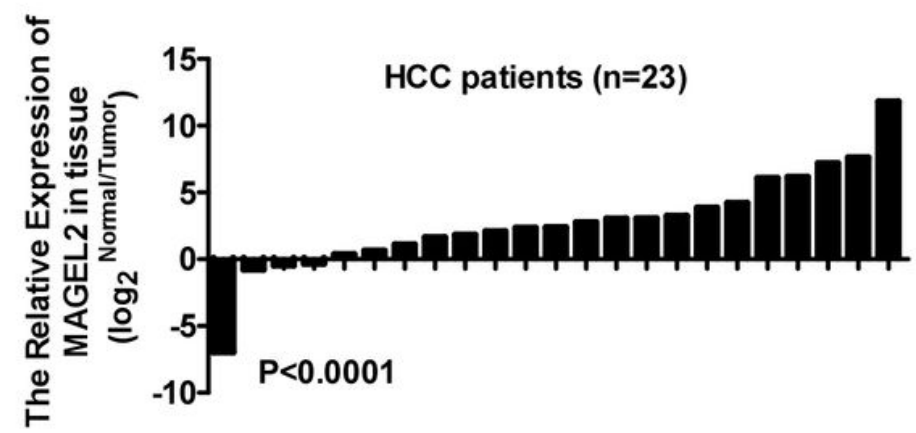

C

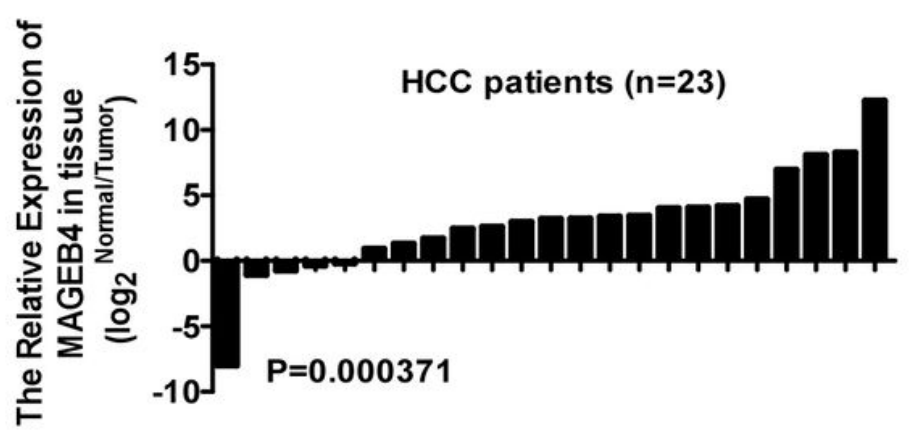

B

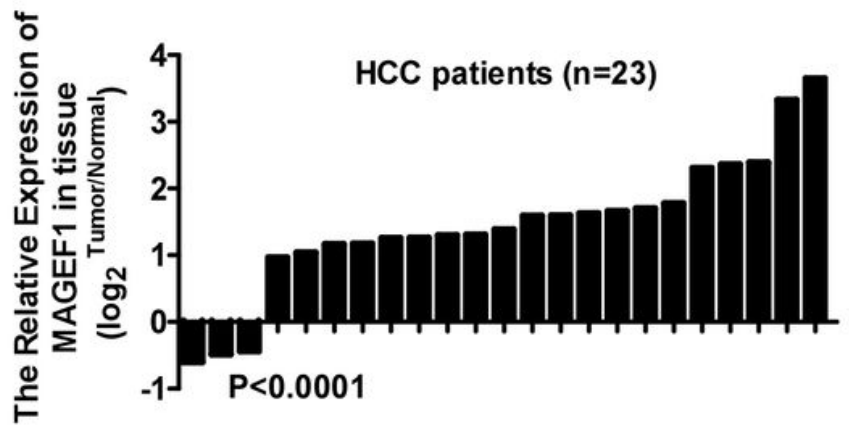

D

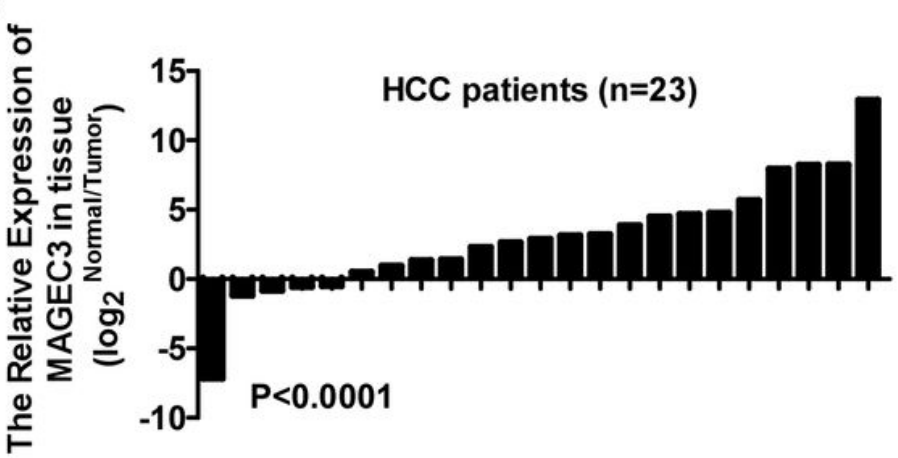

Figure 6

The expression profile of MAGEL2, MAGEF1, MAGEB4 and MAGEC3 in HCC patients of our centre. The qRT-PCR experiment was performed to detect the expression of MAGEs. (A) MAGEL2 was significantly down-regulated in HCC tissues compared with adjacent non-tumor tissues. (B) MAGEF1 was significantly up-regulated in HCC tissues compared with adjacent non-tumor tissues. (C) MAGEB4 was significantly down-regulated in HCC tissues compared with adjacent non-tumor tissues. (D) MAGEC3 was significantly down-regulated in HCC tissues compared with adjacent non-tumor tissues. P values $<0.05$ were considered statistically significant. 

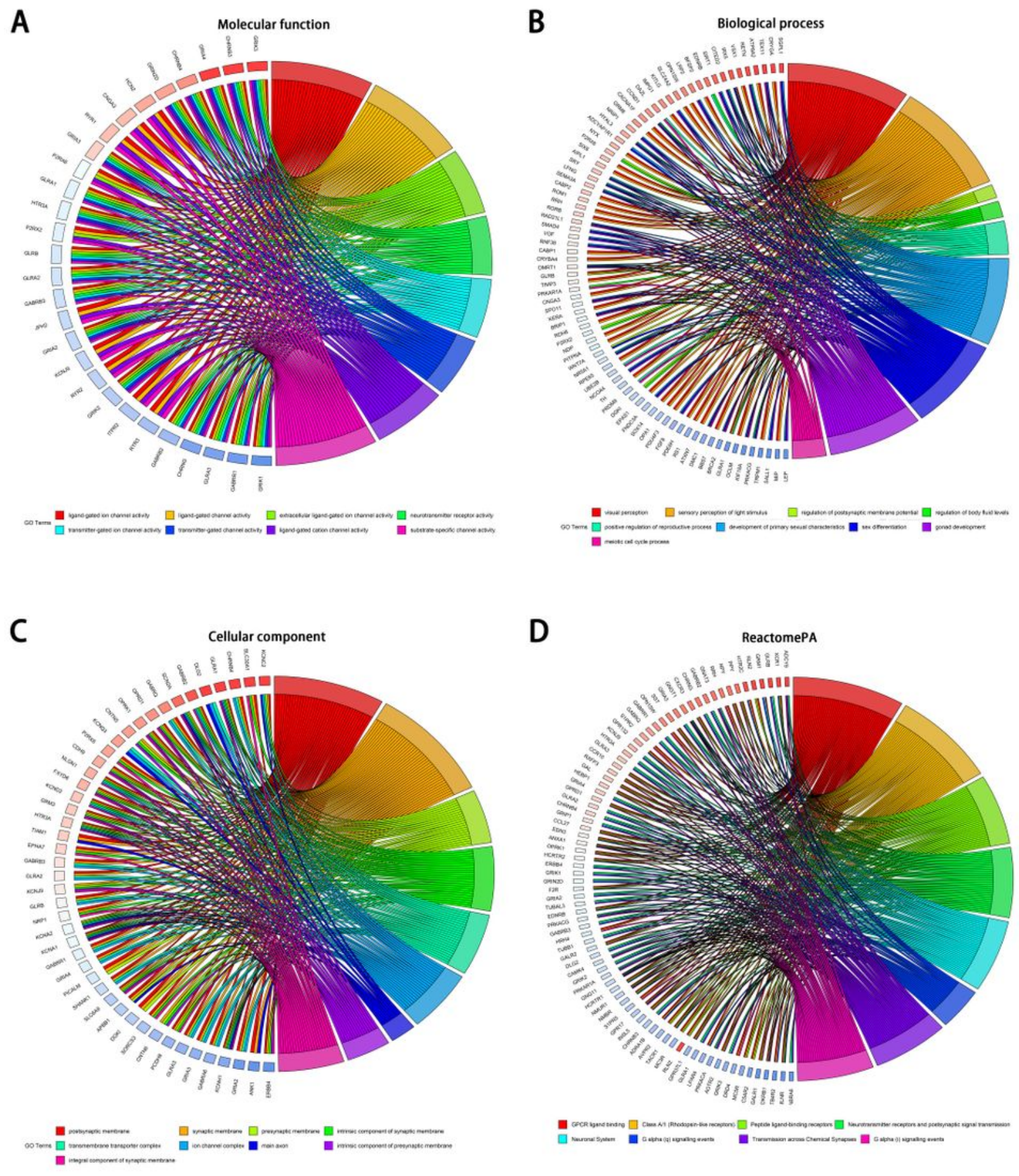

\section{Figure 7}

Functional enrichment analysis of MAGE genes in HCC. The significantly correlated genes with MAGEs in HCC were used to do functional enrichment analysis which were conducted using biomaRt, clusterProfiler, GOplot and ReactomePA packages in R. (A) The Molecular function Circos map showed that the dysregulated MAGEs were closely related to ligand-gated ion channel activity. (B) The Biological process Circos map showed that the dysregulated MAGEs were closely related to cell cycle process and 
developmental process. (C) The Cellular component Circos map showed that the dysregulated MAGEs were closely related to transmembrane transporter complex and ion channel complex. (D) The Reactome pathway Circos map showed that the dysregulated MAGEs were closely related to GPCR ligand binding peptide ligand-binding receptor and $G$ alpha signaling events. $G 0$ terms with $P<0.05$ were considered statistically significant.
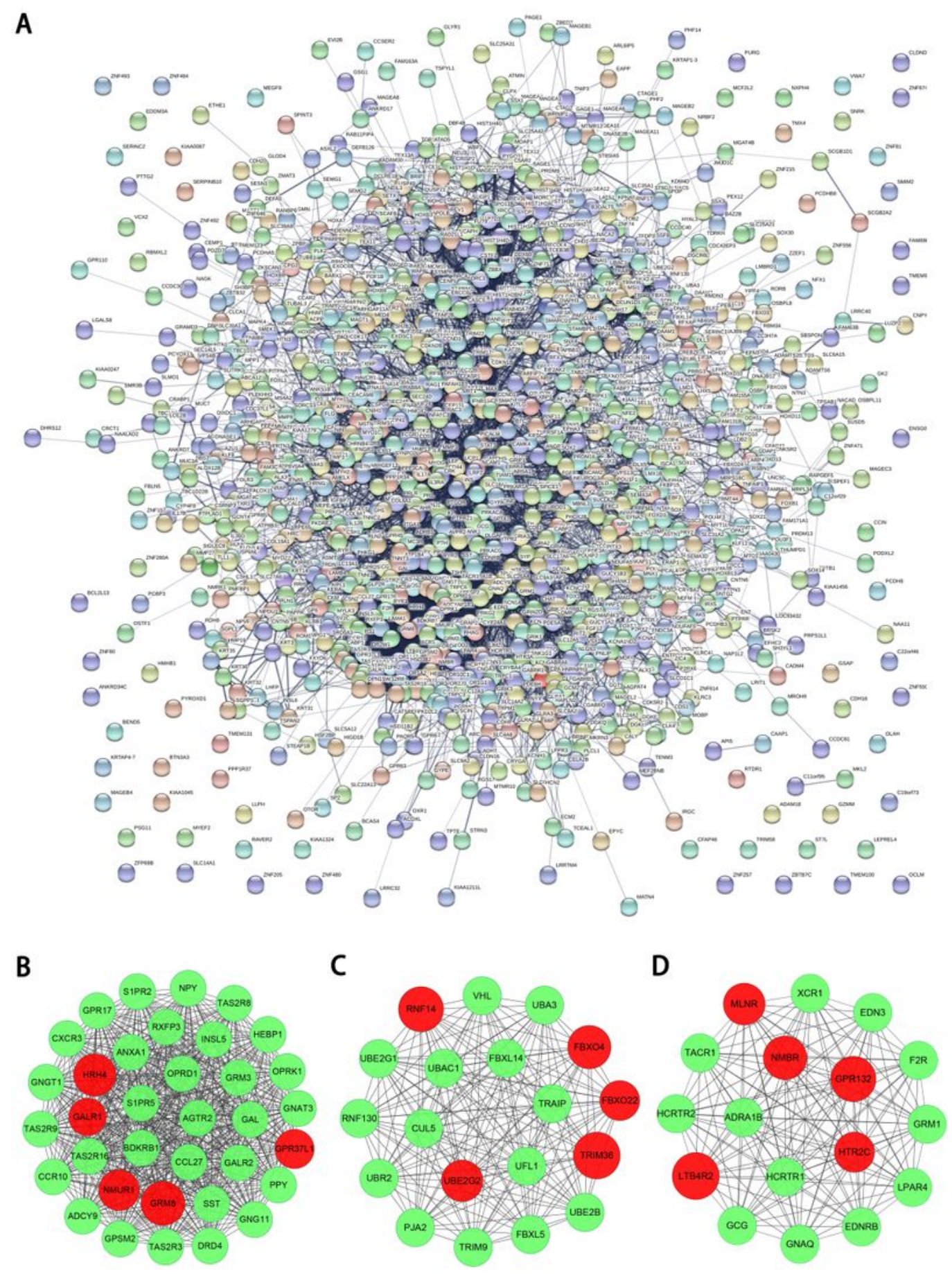

Figure 8 
Protein-protein interaction networks analysis and core genes screening. (A) The protein-protein interaction networks were drawn with STRING and the complex networks showed the interactions between the correlated genes of MAGEs . (B-D) Three major protein interaction regions were isolated with Cytoscape and the top 5 core genes were colored with red. The genes in network 1 (B) were significantly correlated with GPCR ligand binding and signal transduction. The genes in network 2 (C) were significantly correlated with ubiquitin-specific protease activity and regulated cellular protein metabolism. The genes in network 3 (D) were significantly correlated with G-protein coupled activity and the regulation of cellular signal transduction and cell communication.

\section{Supplementary Files}

This is a list of supplementary files associated with this preprint. Click to download.

- tables1.xIsx 